\title{
Dehazing Effects on Image and Video using AHE, CLAHE and Dark Channel Prior
}

\author{
Mahesh Manik Kumbhar, Bhalchandra B. Godbole
}

\begin{abstract}
The image captured by camera is degraded by various atmospheric parameters for example rain, storm, wind, haze, snow. The removing haze is called dehazing, is naturally done in the physical degradation model that requires a resolution of an ill-posed inverse problem. In this paper discussion and e relative study of Adaptive Histogram Equalization (AHE) as well as Contrast limited adaptive histogram equalization (CLAHE) and dark channel prior (DCP). This article suggest image and video dehazing technique working on DCP method. The DCP is resulted from the characteristics of images taken in outdoor that the value of intensity inside the local window is nearly equal to zero. The DCP system has good haze elimination and color managing potential for the images with various angles of haze. The dehazing is done using following four major steps: atmospheric light estimation, transmission map estimation, transmission map refinement, and image reconstruction. This solution of four step DCP will give solution to ill-posed inverse problem. This dehazing techniques can be used in advanced driverless assisted systems in autonomous cars, satellite imaging, underwater imaging etc.

Keywords: Computer vision, image processing, image restoration, image enhancement, dehazing, histogram equalization, dark channel prior.
\end{abstract}

\section{INTRODUCTION} atmospheric elements, the degree of excellence of outdoor images captured in hazy atmosphere circumstances might be ruined brutally [1]. Since degree of excellence loses its quality is related with reduction of contrast and color enhancement products that function consequently with ruined images might not work correctly; such products comprises of multimedia processing systems, automotive real-time vision technologies, surveillance methods, and so on. Hence, to attain good recognition of this type of end user products, the haze in the input hazy image should be erased to improve the degree of excellence of the output dehazed image. Dehazing is the signal processing employed to eliminate haze from the hazy input image [12].

In video surveillance system, the characteristic feature of images is disturbed by the bad weather as like fog, haze and

Revised Manuscript Received on February 05, 2020.

* Correspondence Author

Mahesh Manik Kumbhar*, Department of Electronics and Telecommunication, RIRD, Satara, Shivaji University, Kolhapur, Maharashtra, India. Email: maheshmmkumbhar@gmail.com

Dr. Bhalchandra B. Godbole, Department of Electronics Engineering, KBP, Satara, Maharashtra, India. Email bbgodbole@rediffmail.com

(c) The Authors. Published by Blue Eyes Intelligence Engineering and Sciences Publication (BEIESP). This is an open access article under the CC BY-NC-ND license (http://creativecommons.org/licenses/by-nc-nd/4.0/)
The light reflecting back from object scattered due to

smoke. The efficient defogging technique can increase the functioning of the visual model. So, haze elimination is a challenging issue in computer vision method. The present defogging technique can be separated into two types: one is dependent on the physical type and the other is depend on non-physical type [14].

\section{PHYSICAL HAZE MODEL}

In the haze model, that explains the reduction of atmospheric scene visibility, is a decent estimate for various atmospheric situations and circumstances. Though, it twists the apparent scenes and therefore causes contrary effects on dehazed images at great concentration of fog. Haze model as shown in fig. 2.

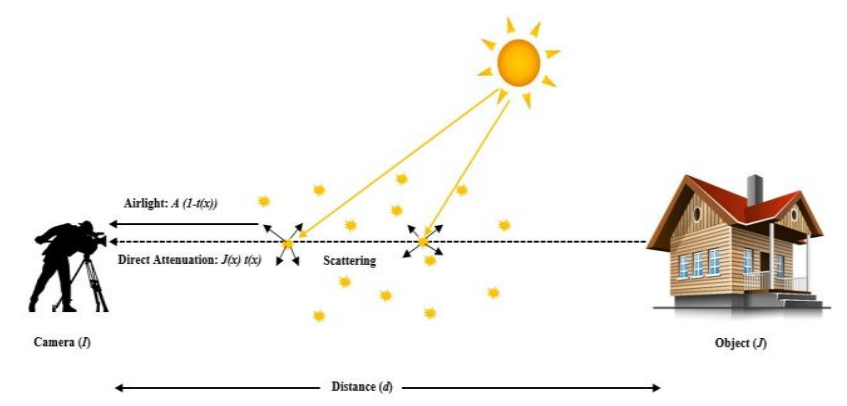

Fig. 1. General Haze Model

The study of the optical reflectance imaging and physical model of haze of atmospheric scattering is done. In Image and video processing, Haze model generally used to explain the construction of a image with haze. Equation (1) is given to attain the Haze model.

$$
I(x)=t(x) J(x)+(1-t(x)) A
$$

Where

$\mathrm{I}(\mathrm{x})$ - hazy image

$\mathrm{J}(\mathrm{x})$ - scene radiance

A - Atmospheric light

$\mathrm{t}(\mathrm{x})(0 \leq \mathrm{t}(\mathrm{x}) \leq 1)$ - scene transmission (color of the image captured by camera).

The man aim of removing haze is to get original value of the $\mathrm{J}(\mathrm{x}), \mathrm{A}$, and $\mathrm{t}(\mathrm{x})$ by using $\mathrm{I}(\mathrm{x})$.

This model in equation (1) clarifies the loss of contrasts because of haze in the image as the outcome of averaging the image with a persistent color A. If the contrasts measured in the image as the magnitude of its gradient field, a scene $\mathrm{J}$ perceived over a uniform medium with $\mathrm{t}(\mathrm{x})=\mathrm{t}<1$ gives us $\|\nabla \mathrm{II}\|=\|\mathrm{tVJ}(\mathrm{x})+(1-\mathrm{t}) \nabla \mathrm{AA}\|=\mathrm{t}\|\mathrm{VJ}(\mathrm{x})\|<\|\mathrm{VJ}(\mathrm{x})\|$ (2)

Where 
$\nabla$ - gradient value of the image

Since $0 \leq t(x) \leq 1$, above formula shows that, As the average gradient value of the hazy image minimizes, the haze in the image increases, producing in a hazy image.

For a K-pixel color image I(x), there are $3 \mathrm{~K}$ constraints and $4 K+3$ unknowns. So that, it is challenging to get a Haze free image from a current hazy image, as the unknowns are belongings of the haze-free image. In equation (1), the first term $t(x) J(x)$ is called as the direct attenuation, it is related to transmission coefficient. The $(1-t(x)) A$ term is called the airlight. Direct attenuation explains the scene radiance, and shrinks with $\mathrm{t}(\mathrm{x})$. As $\mathrm{t}(\mathrm{x})$ falls the airlight increases. The main cause for the falling of image quality is that the scene radiance, it is washed-out by haze and far-away objects. If the haze in the image is consistent, then $\mathrm{t}(\mathrm{x})$ is written as

$$
t(x)=e^{-\beta d(x)}
$$

Where

$\mathrm{d}(\mathrm{x})$ - scene depth

$\beta$ - medium extinction coefficient.

Geometrically, as per equation (1), In the RGB color space the haze model equation shows that, A, I (x) and $\mathrm{J}(\mathrm{x})$ are vector coplanar and their termination points are collinear. Two line segments ratio is the transmission map $\mathrm{t}(\mathrm{x})$, ie.

$$
t(x)=\frac{\|[A-\eta[x) \|}{\|A-\jmath(x)\|]}=\frac{A^{2}-l^{2}[(x)}{A^{2}-\jmath^{2}(x)}
$$

Where

c e $\{R, G, B\}$ - color channel index

\section{DEHAZING METHODOLOGIES}

The technique depend on physical system uses the atmospheric scattering system to restore the image by getting inverse process of image makes to lose its quality, whereas the technique depend on non-physical type don't test physical reason of image losing its quality produced by the weather parameters, it is a subjective process and aims at improving image degree of excellence according to the visual perception [16]. Generally the technique depend on physical type is efficient than non-physical type on the effect of haze elimination. The trending concept of haze removal technique is the technique depend on physical type in recent years [2], [19].Technique depend on the dark channel prior (DCP) is simple, but it do efficient dehazing. The DCP-depend dehazing technique shows impressive dehazing degree of excellence when compared to other previous methods [3].

Fewer quantity of research only has been completed in image processing of un-degree of excellence images associated with the other image processing branches. IE is primarily increasing the image degree of excellence without any loss of information. Enhancement methods are product specific and accordingly, for a particular product, certain features that are needed to be focused are enhanced [4].Alternatively, the video saliency type gets less attention compared to the images, but it has obtained good consideration nowadays. Distinct from image saliency identification, motion data offers a strong prior [5].

Since night time image had its light not mono-color (traffic lights), and was not like daytime image having sunlight regularly glowing on, the area with color light irradiation did not shield the whole picture, and may be one or several neighboring areas [6]. This article, presents a real-time image enhancement approach using an improved dark channel prior type depend on atmospheric scattering nature [7]. Repetitious take out Transmission beneath the theory that large-scale chromaticity changes are because of Transmission while small-scale luminance varies [8].

In this article section II shows the haze model, section III will give us existing related work, section IV describes the Experimental results, analysis and comparison, section $\mathrm{V}$ concludes all the work.

Previously popularly utilized technique are as following:

A. AHE - Adaptive Histogram Equalization

B. CLAHE- Contrast limited adaptive histogram equalization

\section{C.DCP- Dark Chanel Prior}

\section{A. Adaptive Histogram Equalization (AHE)}

Adaptive Histogram Equalization (AHE) technique is the improved work of histogram equalization technique. In AHE technique enhancement practices are put on over a explicit area of an image and fine-tune contrast rendering to their adjacent pixels. AHE is used for improvement in HSV color space. 'adapthisteq' function applied in MATLAB for equalization of intensity as per the adjacent pixels. This technique is utilized only for homogeneous haze correction, For enhancement in image Dynamic range separation Histogram equalization (DRSHE) technique is used. In DRSHE technique histogram separated in some pre-set sections. Then alter the brightness of that same region and regularly share into gray scale image. The main drawbacks of AHE technique is the potential to over-intensify noise in comparatively consistent haze areas of an image [10].

\section{B. Contrast Limited Adaptive Equalization (CLAHE)}

Contrast limited adaptive equalization is a customized concept of AHE. Augmentation function is executed on the entire adjacent pixels and transformation process is extracted in CLAHE method. This varies from AHE because of its restriction in contrast. CLAHE technique for improvement process, in this technique utilized greatest value to alter the histogram and reorganize in gray level image. This technique implemented individually for background and foreground. This technique also limits the noise, recovers the contrast from hazy image. 'Rayleigh' distribution factors are utilized for bell shaped histogram. CLAHE executed on the two varieties of images namely gray scale and color. 'Clip limit' function is utilized for put on bound on a noise image. Color space is utilized for RGB true color images. The main drawback is CLAHE functions on tiny data regions (tiles), instead on the whole image. It is computationally more cost. It is quite complicated in hardware [10].

\section{Dark Channel Prior (DCP)}

Dark Channel Prior (DCP) technique is developed for the enhancement of image and video quality. DCP was proposed in recent times and has obtained a huge acceptance. The DCP is extracted from feature of normal outdoor images that the brightness value of minimum one color channel within a local window is close to zero.

Published By:

Retrieval Number: C4833029320/2020@BEIESP 
Depending on the DCP, the dehazing is carried out using following key stages: atmospheric light calculation, Transmission map calculation, Transmission map modification, and original image restoration. Above four-stage dehazing function makes it feasible to offer a stage-by-stage method to the complex solution of the ill-posed inverse issue [15]. The method furthermore provides to get rid of light on the regular assistance of modern researches associated to the DCP for all stages of dehazing function. This broad review as well as experimental testing on DCP-related techniques will assist to recognize the efficiency of the single stage of the dehazing function and will smooth the progress of growth of superior dehazing methods [2].

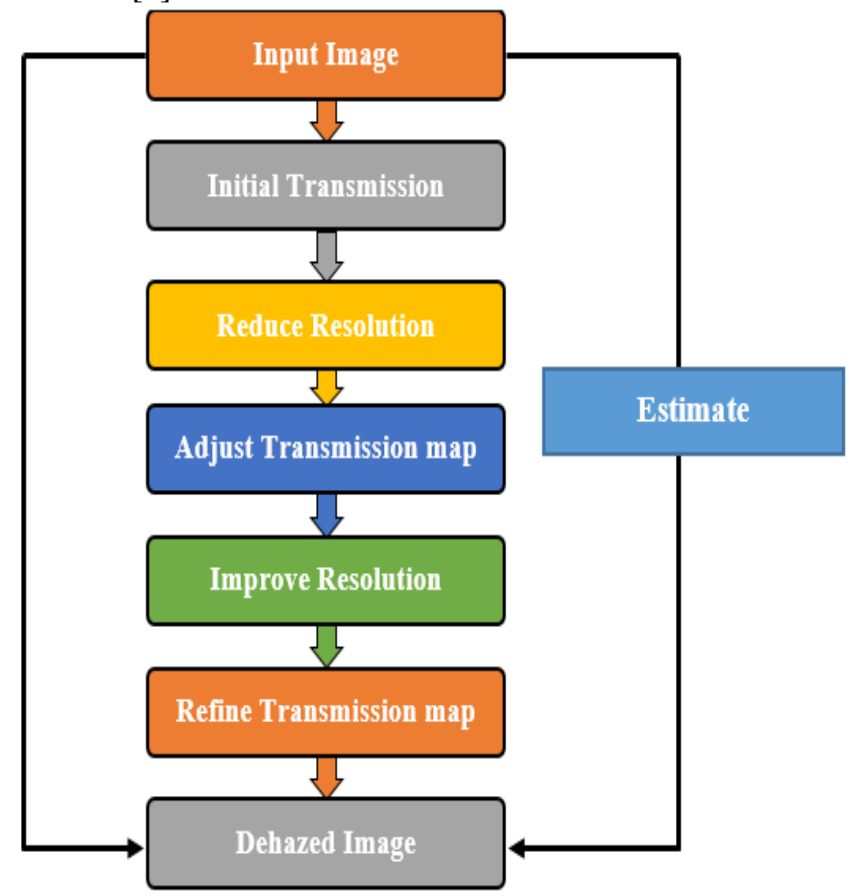

Fig. 2. Image Enhancement Using DCP

In certain fog images, which has more of sky regions or huge bright regions, the image accepted the technique of dark channel prior defogging will emerge as color deformation. This happens because the dark channel value on these regions is hard to near to zero, even supposing with no fog. So the theory of dark channel prior is not appropriate for these regions. Additionally, in order to increase the functioning rate of algorithm, the concept of multi-resolution is utilized to reduce the pixel intensities of early Transmission rate map. Subsequently, the technique is tuned to reduce calculation time. Flow chart of the algorithm is displayed as fig. 2.

\section{EXPERIMENTAL RESULTS AND ANALYSIS}

The proposed algorithms is implemented on a Windows 10 PC with an Intel (R) Core (TM) i5-4210U CPU@2.40 $\mathrm{GHz}$ processor, running Matlab 2019b. We have taken 200+ images taken from internet open google image library, also own captured pictures. The set of experimental images are classified as traffic images, building images along with sky, vehicle images and natural images.

Fig. 3. shows the original input hazy image. A set of hazy images are taken, dehazing consists of the technique depending on the AHE, CLAHE, DCP. In DCP it provides the initial Transmission map.

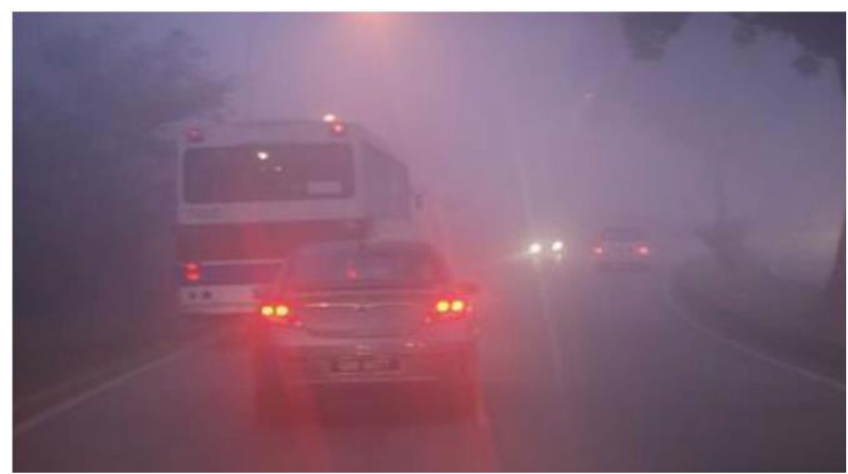

Fig. 3. Original Hazy input image

This prior originates from the remark that most non- sky patches in outdoor haze-free images have minimum one color channel with few low intensity pixels. The intensity of these dark pixels is mostly because of the airlight in hazy images Natural outdoor images are normally having shadows. These images produce dark channel as very dark. The dark channel contains so many bright pixels they are mostly a part of sky or haze. Brightness of dark channel is higher because of haze and fog present in scene. The haze extent of the in input image can be estimated by using the intensity of the dark channel. Fig. 4. Displays their corresponding dark channel images

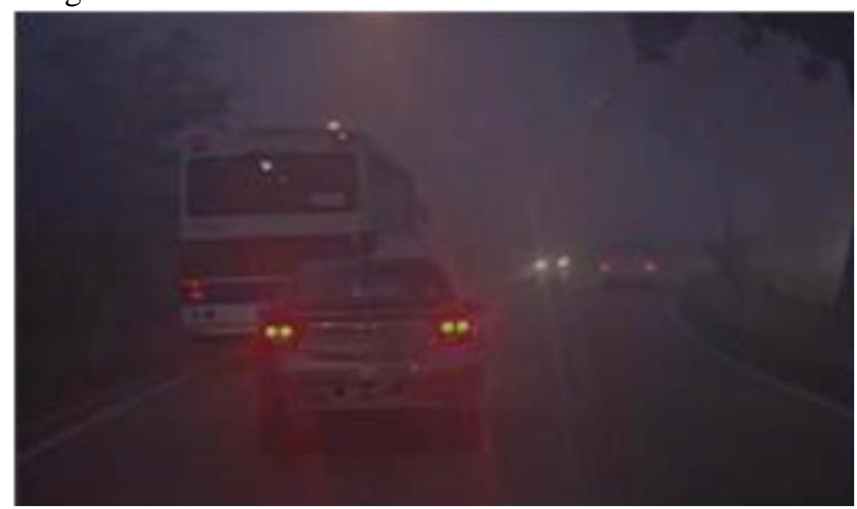

Fig. 4. Dark Channel

As displayed in Fig. 5, color deviation occurs in the dealing of the brightness area through dark channel prior, specifically in the sky area which has abnormal color spreading beyond its border. In fig. 5. It shows that the sky and empty regions gives the zero transmission. In hazy atmosphere we lost ability to see the depth in the image. We refine this transmission map by using soft matting method.

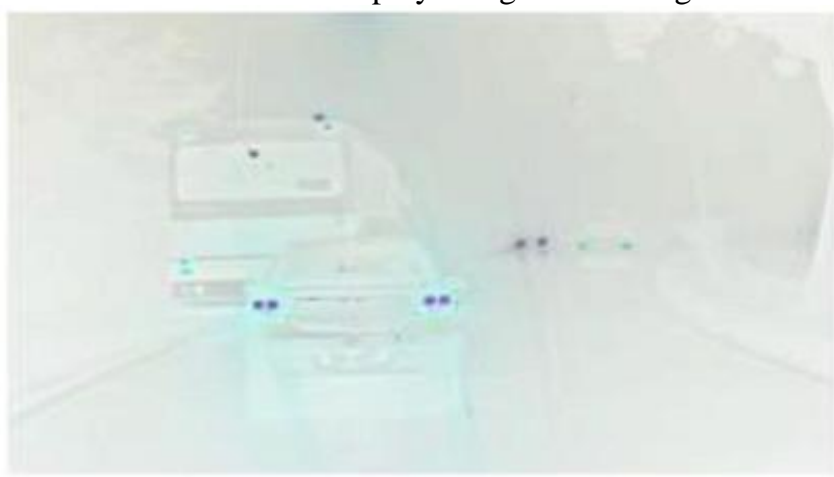

Fig. 5. Transmission map

Published By:

Blue Eyes Intelligence Engineering

Retrieval Number: C4833029320/2020(CBEIESP

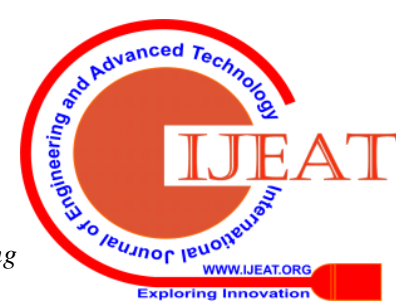


In fig. 6 it is seen that refined transmission map achieves to get the sharp edge discontinuities as well as sketches the profile of the objects in transmission map. The difference in transmission map and refined transmission map can be observed in fig. 5 and 6.

Fig. 6. Refined Transmission map

The color of the dehazed image obtained by the DCP is more normal, and very consistent with the original. When introducing the AHE and CLAHE method, the brightness and contrast of the image got changed and abnormal.

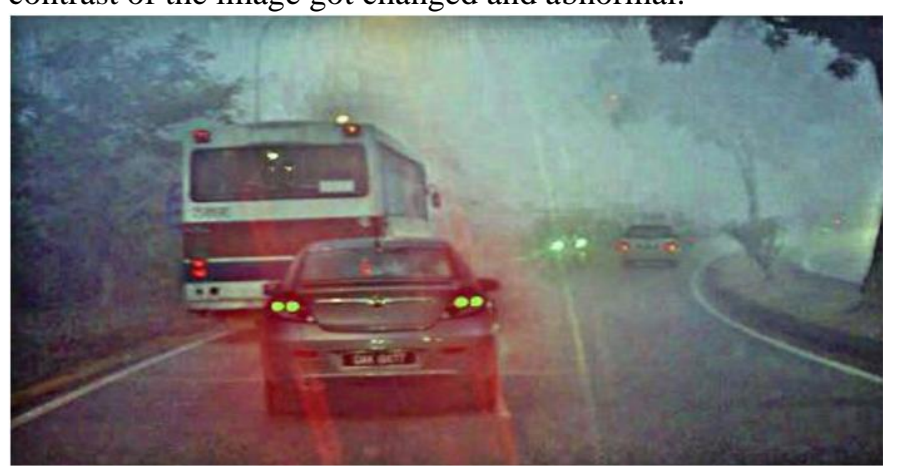

Comparison of existing image dehazing algorithms ie. AHE, CLAHE and DCP. In APPENDIX fig. 9 a) is the original Hazy Input Image. When the CLAHE is applied to the hazy image the resultant output image is shown in fig. $9 \mathrm{~b}$ ). In the CLAHE image colors had an effect by shine and chromatic deviation, are observed so that images are badly damaged. For enhanced result, DCP considers the region disturbed by shine, so that shine is not made improved, but blocking manmade objects are enormously clear in its improved result, after applying the DCP technique of this paper its output is shown in Fig. 9. c), in which the issue of preventing manmade objects is enhanced, building the image restoration to be further normal [9]. For the method comparison using different types of images used. The images we have taken from internet source using the process. Compare the existing and proposed system, and compute the PSNR (Peak signal to noise ratio), MSE (Mean Square Error), Entropy (Energy of the image), Correlation (similarity measure). All parameters are shown in Table 1 and compared in Fig. 8 (a-d)

Fig. 7. Dehazed Image

Table 1. Output Parameters Comparisons

\begin{tabular}{|c|c|c|c|c|c|c|c|c|}
\hline Parameters & \multicolumn{2}{|c|}{ PSNR } & \multicolumn{2}{c|}{ MSE } & \multicolumn{2}{c|}{ ENTROPY } & \multicolumn{2}{c|}{ CORRELATION } \\
\hline Image name & CLAHE & DCP & CLAHE & DCP & CLAHE & DCP & CLAHE & DCP \\
\hline Forest & 15.481 & 68.67 & $4.5 \mathrm{e}+03$ & $1.4 \mathrm{e}+03$ & 6.52 & 6.83 & 0.82 & 0.98 \\
\hline Fort & 19.762 & 69.9 & $4.9 \mathrm{e}+03$ & $1.2 \mathrm{e}+03$ & 6.11 & 6.72 & 0.8 & 0.962 \\
\hline Pumpkin & 19.4 & 69 & $4.23 \mathrm{e}+03$ & $1.42 \mathrm{e}+03$ & 6.02 & 6.99 & 0.832 & 0.941 \\
\hline Train & 18.9 & 61.2 & $4.76 \mathrm{e}+03$ & $1.39 \mathrm{e}+03$ & 5.92 & 6.86 & 0.813 & 0.927 \\
\hline Vehicle & 16.92 & 68.9 & $4.40 \mathrm{e}+03$ & $1.1 \mathrm{e}+03$ & 6.06 & 6.78 & 0.847 & 0.948 \\
\hline
\end{tabular}

\section{A. Peak signal-to-noise ratio (PSNR)}

PSNR is the ratio of the maximum possible power of a signal to the power of mortifying noise that affects the reliability of its illustration as shown in Fig. 8 (a). PSNR is normally uttered in logarithmic decibel scale as the various signals having wide dynamic range represented in equation 5

$$
\text { PSNR }=10 . \log _{10} \cdot\left(\frac{\operatorname{MAX}_{i}^{2}}{M S E}\right)
$$

$\mathrm{MAX}_{I}^{2}$-Maximum square value of pixel in the image

As DCP is having lager value of PSNR than the CLAHE it can be said that the in DCP the reconstructed dehazed image quality is better than CLAHE.

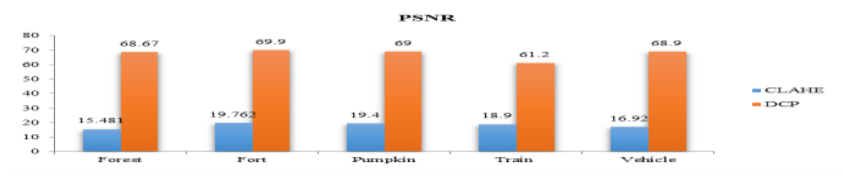

Fig. 8. a) PSNR Comparison of CLAHE and DCP

\section{B. Mean squared error (MSE):}

MSE is given by Equation (6). The inverse relationship between PSNR and MSE is there so As DCP is having lower value of MSE than the CLAHE it can be said that the in DCP the reconstructed dehazed image quality is better than CLAHE

$$
\text { MSE } \left.=\frac{1}{M N} \sum_{n=1}^{M} \sum_{m=1}^{N}[\text { [INPUT }- \text { OUTPUT })\right]^{2}
$$


MSE

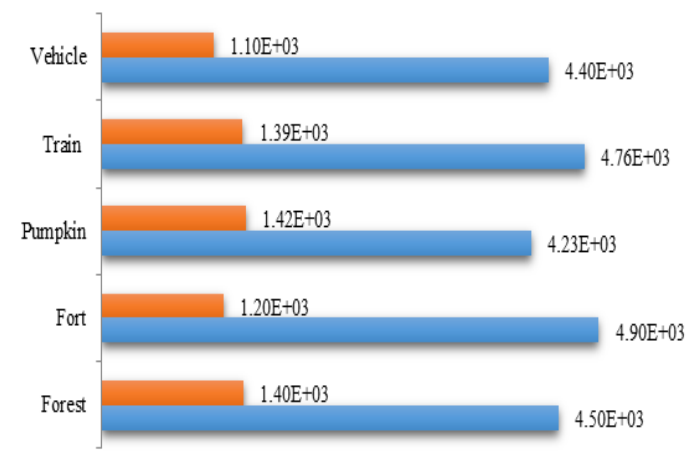

Fig. 8. b) MSE Comparison of CLAHE and DCP

\section{Correlation}

Figure 8 (c) is The correlation coefficient that indicates the strength of the relationship between two images(input and output) can be found in Equation (7),

(7)

$$
r_{X Y}=\frac{\sum\left(X-X_{i}\right)\left(Y-Y_{i}\right)}{\sqrt{\sum(X-X i)^{2}\left(Y-Y^{i}\right)^{2}}}
$$

\section{$r_{X Y}$ - Correlation}

X-Image Row Pixel, Y- image Column pixel

$\mathrm{X}, \mathrm{Y}$ - Input

$\mathrm{Xi}, \mathrm{Yi}$ - output Image

Correlation is more slightly ore in DCP as compared to CLAHE. So the DCP give us more similar image as input to output.

\section{Correlation}

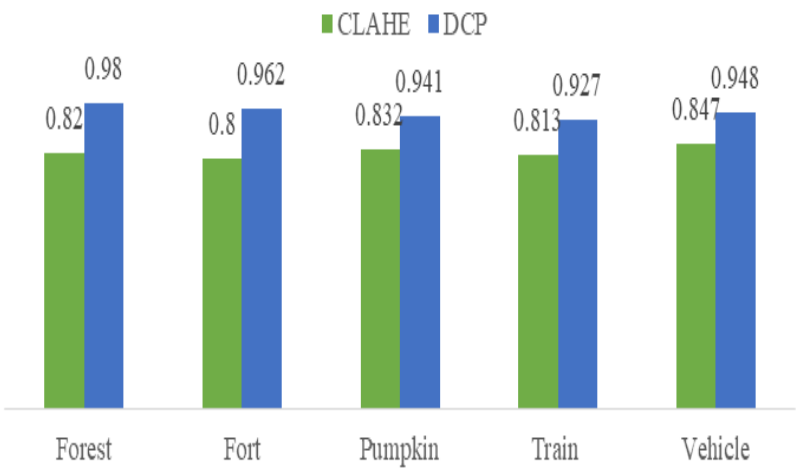

Fig. 8. c) Correlation Comparison of CLAHE and DCP

\section{Entropy}

Figure 8 (d) is statistical degree of randomness that can be used to characterize the texture of the input image. Equation (8) is given by

$$
\text { Entropy }=\Sigma\left(\text { p. }{ }^{*} \log _{2}(\mathrm{p})\right)
$$

p - Counts normalized histogram

As seen in graph entropy is more in DCP as compared to CLAHE

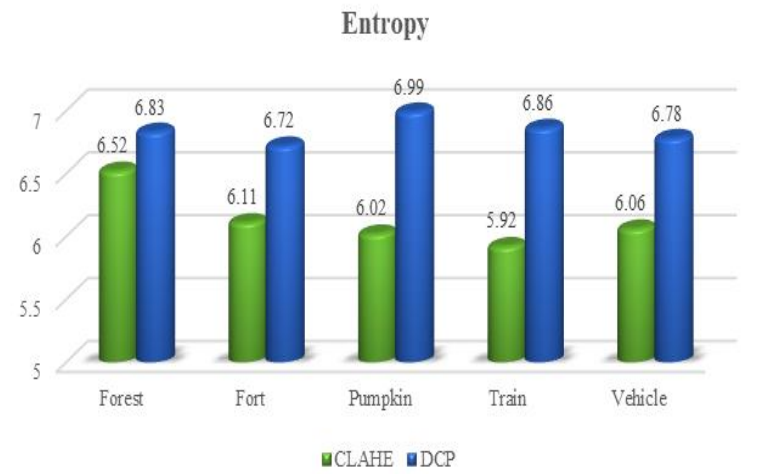

Fig. 8. d) Entropy Comparison of CLAHE and DCP

\section{CONCLUSION}

This paper provides a broad comparative study of the best effective dehazing techniques. The image dehazing using DCP technique along with AHE and CLAHE. There are four essential steps of the DCP image dehazing, which are atmospheric light estimation, transmission map estimation, transmission map refinement, and image reconstruction. Conventional methods AHE and CLAHE are conducted step-by-step analysis and experiments. Additionally, the conventional methods implemented on Matlab for assessing the recital of image dehazing is also brief and conversed. The quality of the image and video is improved a lot and the values of PSNR are found as high for DCP than the CLAHE and the value of MSE is found less in DCP. We are sure that our in depth survey and experimental analysis will help future developments to comprehend the DCP based dehazing methods and also will assist in development of progressive dehazing algorithms. 
Dehazing Effects on Image and Video using AHE, CLAHE and Dark Channel Prior

APPENDIX
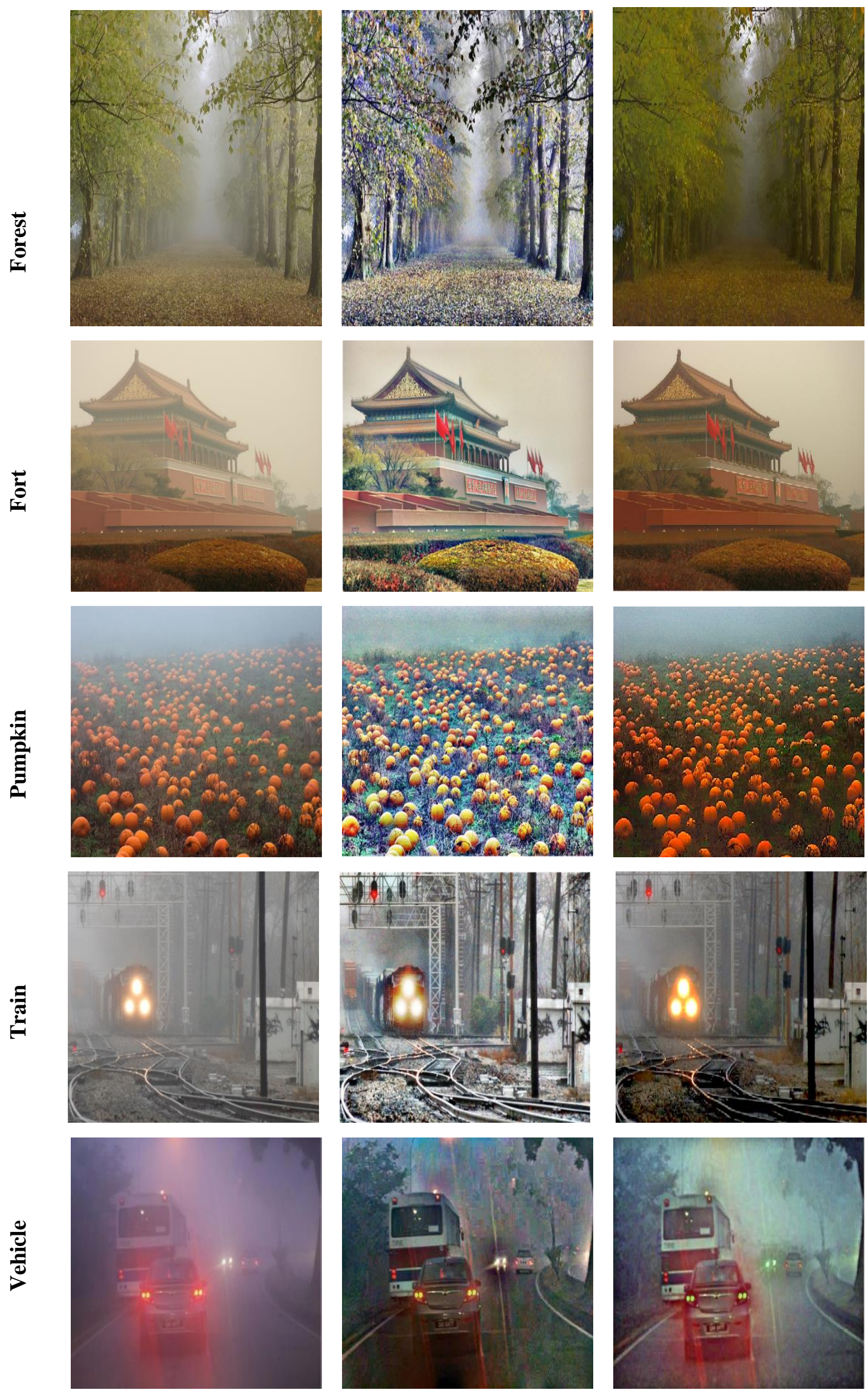

Fig. 9 a) Hazy Image

b) CLAHE Output

c) DCP output

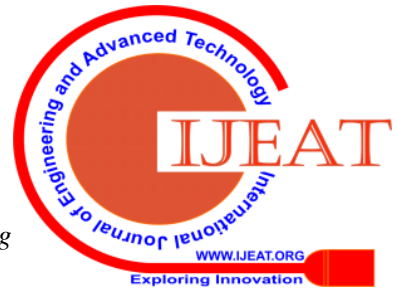




\section{REFERENCES}

1. Yongmin Park, Tae-Hwan Kim, "Fast Execution Schemes for Dark-Channel-Prior-Based Outdoor Video Dehazing", IEEE Access, Volume 6, 2018.

2 E. Zhang, K. Lv, Y. Li and J. Duan, "A fast video image defogging algorithm based on dark channel prior," 2013 6th International Congress on Image and Signal Processing (CISP), Hangzhou, 2013, pp. 219-223.

3. Park, Yongmin and Tae-Hwan Kim. "A video dehazing system based on fast airlight estimation.” 2017 IEEE Global Conference on Signal and Information Processing (GlobalSIP) (2017): 779-783.

4. S. Dixit, S. K. Tiwari and P. Sharma, "Underwater image enhancement using DCP with ACCLAHE and homomorphism filtering," 2016 International Conference on Signal Processing, Communication, Power and Embedded System (SCOPES), Paralakhemundi, 2016, pp. 2042-2046.

5. K. Tian, Z. Lu, Q. Liao and N. Wang, "Video saliency detection based on robust seeds generation and spatio-temporal propagation," 2017 10th International Congress on Image and Signal Processing, BioMedical Engineering and Informatics (CISP-BMEI), Shanghai, 2017, pp. 1-6.

6. J. R. Wang, C. H. Su and J. Y. Chiang, "SmartCam to see through darkness," 2016 IEEE International Conference on Systems, Man, and Cybernetics (SMC), Budapest, 2016, pp. 003234-003239.

7. X. Jiang, H. Yao, S. Zhang, X. Lu and W. Zeng, "Night video enhancement using improved dark channel prior," 2013 IEEE International Conference on Image Processing, Melbourne, VIC, 2013, pp. 553-557

8. Yeh, Chia-Hung \& Kang, Li-Wei \& Lin, Cheng-Yang \& Lin, Chih-Yang."Efficient image/video dehazing through haze density analysis based on pixel-based dark channel prior", Proceedings - 3rd International Conference on Information Security and Intelligent Control, ISIC 2012. 238-241.

9. Das, Dibyasree et al. "Dehazing technique based on dark channel prior model with sky masking and its quantitative analysis." 2016 2nd International Conference on Control, Instrumentation, Energy \& Communication (CIEC) (2016): 207-210.

10. Yadav, Garima et al. "Contrast limited adaptive histogram equalization based enhancement for real time video system." 2014 International Conference on Advances in Computing, Communications and Informatics (ICACCI) (2014): 2392-2397.

11. Lee, S., Yun, S., Nam, JH. et al, "A review on dark channel prior based image dehazing algorithms", EURASIP Journal on Image and Video Processing volume 2016, 2016:4.

12 R. Sathya, M. Bharathi and G. Dhivyasri, "Underwater image enhancement by dark channel prior," 2015 2nd International Conference on Electronics and Communication Systems (ICECS), Coimbatore, 2015, pp. 1119-1123.

13. Tanghuai Fan ; Xiao Ma ; Zhen Zhang ; Hongxin Wu, "An improved image defogging method based on dark channel prior", 2nd International Conference on Image, Vision and Computing (ICIVC), 2017.

14. Sungmin Lee, Seokmin Yun, Ju-Hun Nam, Chee Sun Won, "A review on dark channel prior based image dehazing algorithms", EURASIP Journal on Image and Video Processing , 2016.

15. Sebastian Salazar-Colores, Juan-Manuel Ramos-Arreguín, Jesus-Carlos Pedraza-Ortega, J Rodríguez-Reséndiz, "Efficient single image dehazing by modifying the dark channel prior", EURASIP Journal on Image and Video Processing volume 2019.

16. Xiuyuan Wang, Chenghai Yang, Jian Zhang, Huaibo Song, "Image dehazing based on dark channel prior and brightness enhancement for agricultural monitoring", International Journal of Agricultural and Biological Engineering, 2018.

17. Chengyi Cai; Yiheng Zhang; Ting Liu, "Underwater Image Processing System for Image Enhancement and Restoration", IEEE 11th International Conference on Transmission Software and Networks (ICCSN), 2019.

18. Jehoiada Jackson; Oluwasanmi Ariyo, "Hybrid single image dehazing with bright channel and dark channel priors", 2nd International Conference on Image, Vision and Computing (ICIVC), 2017.

19. Yutaro Iwamoto; Naoaki Hashimoto, "Fast Dark Channel Prior Based Haze Removal from a Single Image", 14th -ICNC-FSKD, 2018.

20. Guoling Bi ; Jianyue Ren ; Tianjiao Fu ; Ting Nie ; Changzheng Chen ; Nan Zhang, " Image Dehazing Based on Accurate Estimation of Transmission in the Atmospheric Scattering Model", IEEE Photonics Journal, 2017.
21. Lee, S., Yun, S., Nam, JH. et al., "A review on dark channel prior based image dehazing algorithms", EURASIP Journal on Image and Video Processing, December (2016) 2016: 4.

\section{AUTHORS PROFILE}

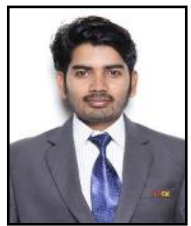

Mahesh Manik Kumbhar, is Research Scholar at RIRD, Satara under Shivaji University, Kolhapur, MH, India. His area of research is Image and Video Processing. He has total 9years of teaching experience as Assistant Professor and having 6 months of industrial experience. He has published 15 plus research papers and one book.

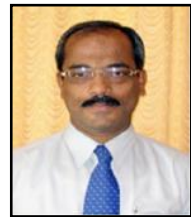

Dr. Bhalchnadra B. Godbole is working as Associate Professor at KBP, Satara, MH, India. His area of research is Signal and Image Processing. He has total 30 years of teaching experience. He has also worked as Board of Studies Chairman in Shivaji University, Kolhapur. He has published 40 plus research papers and one book. 